\title{
Bayesian Joint Inversion Implies a Complex Multiscale Lowermost Mantle Overlaying Simple Core- Mantle Boundary Topography
}

\author{
JACK B MUIR ${ }^{1,2}$, SATORU TANAKA ${ }^{3}$ AND HRVOJE \\ TKALČIĆ 2 \\ ${ }^{1}$ California Institute of Technology \\ ${ }^{2}$ Australian National University \\ ${ }^{3}$ D-EARTH, JAMSTEC \\ Presenting Author: jmuir@caltech.edu
}

A combined understanding of both the velocity structures of the Lowermost Mantle (LM) and the underlying topography of the Core-Mantle Boundary (CMB) is an essential input into models of the dynamics of the deep Earth. While long-period tomographic Vs models of the lowermost mantle have begun to agree in recent years, there is poorer agreement between $\mathrm{Vp}$ models and even less agreement between models of CMB topography, which reflects the sparser sampling and poor sensitivity of most seismic observations to CMB topography, as well as the tradeoff between velocity in the LM and CMB topography. In order to better resolve both of these features, we utilize a joint inversion of LM velocity and CMB topography using a meticulously curated hand-picked dataset of PcP-P, $\mathrm{PKPab}-\mathrm{PKPbc}$ and $\mathrm{P} 4 \mathrm{KP}-\mathrm{PcP}$ differential travel time phases. This collection features short-period phases both in CMB reflection and transmission, allowing both velocity and topography to be independently controlled. We utilize hierarchical Hamiltonian Monte Carlo sampling to marginalize over the unknown data error and a priori characteristic size of velocity and topography perturbations, and posterior-predictive cross-validation to determine the highest model resolution that may be supported by the data. We find that the perturbation spectrum of the velocity spectrum is broadband, supporting observations of multiscale thermally dominated features in the LM, while the topography is roughly hemispherical with the spectrum concentrated at lower spherical harmonic degrees. The disconnect between these two spectra suggest that CMB topography is not heavily influenced by isostasy but is instead driven by dynamic topography, with the amplitude of the observed topography not requiring a low viscosity channel in the LM. 